\title{
ON FUZZY ORDERED LA-SEMIHYPERGROUPS
}

\author{
MUHAMMAD AZHAR ${ }^{1}$, MUHAMMAD GULISTAN ${ }^{1, *}$, NAVEED YAQOOB $^{2}$, SEIFEDINE KADRY $^{3}$ \\ ${ }^{1}$ Department of Mathematics, Hazara University, Mansehra, KP, Pakistan \\ ${ }^{2}$ Department of Mathematics, College of Science Al-Zulf, Majmaah University, Al-Zulfi, Saudi Arabia \\ ${ }^{3}$ Department of Mathematics \& Computer Science, Beirut Arab University, Lebanon \\ ${ }^{*}$ Corresponding author: gulistanmath@hu.edu.pk
}

\begin{abstract}
We introduce the notion of fuzzy ordered LA-semihypergroups and provide different examples.
\end{abstract}
We also discuss some results related with fuzzy left and right hyperideals.

\section{Introduction}

The theory of algebraic hyperstructure was introduced by Marty in 1934, when Marty [1] defined hypergroups. Since then many hyperstructures were being studied by several authors, for instance, Bonansinga and Corsini [2], Davvaz [3], Freni [4], Hila et al. [5], Leoreanu [6], Salvo et al. [7] and many others. The concept of ordered semihypergroup was studied by Heidari and Davvaz in [8], where they used a binary relation $" \leq$ " on semihypergroup $(H, \circ)$ such that the binary relation is a partial order and the structure $(H, \circ, \leq)$ is known as ordered semihypergroup. There are several authors who study the ordering of hyperstructures, for instance, Bakhshi and Borzooei [9], Chvalina [10], Hoskova [11], Kondo and Lekkoksung [12] and Novak [13].

Another non-associative algebraic hyperstructure known as LA-semihypergroup which is a useful generalization of semigroup, semihypergroups and LA-semigroups was introduced by Hilla and Dine [14] in 2011 based on left invertive law given by Kazim and Naseerudin [15] in 1972. Yaqoob et al. [16] extended the work

Received 2017-12-07; accepted 2018-02-01; published 2018-03-07.

2000 Mathematics Subject Classification. 20N20.

Key words and phrases. LA-semihypergroups; ordered LA-semihypergroups; fuzzy hyperideals.

(c)2018 Authors retain the copyrights of their papers, and all open access articles are distributed under the terms of the Creative Commons Attribution License. 
of Hila and Dine and characterized intra-regular left almost semihypergroups by their hyperideals using pure left identity. The ordering in LA-semihypergroups was introduced by Yaqoob and Gulistan [17].

The concept of fuzzy set was introduced by Zadeh in 1965 [18]. Rosenfeld [19] introduced fuzzy sets in the context of group theory and formulated the concept of fuzzy subgroup of a group in 1971. Later many researcher are engaged in extending the concept of abstract algebra to the frame work of fuzzy setting. Fuzzy hyperstructures have been already considered by many researchers, for instance, Corsini et al. [20,21], Davvaz [22, 23], Hila and Abdullah [24], Khan et al. [25], Pibaljommee et al. [26, 27], Tang et al. [28-31], Tipachot and Pibaljommee [32] and Zhan et al. [33,34].

As a further study of ordered LA-semihypergroups, we attempt in the present paper to study the fuzzy ordered LA-semihypergroups in detail.

\section{Preliminaries}

Let $H$ be a non-empty set. Then the map $\circ: H \times H \rightarrow \mathcal{P}^{*}(H)$ is called hyperoperation or join operation on the set $H$, where $\mathcal{P}^{*}(H)=\mathcal{P}(H) \backslash\{\emptyset\}$ denotes the set of all non-empty subsets of $H$. A hypergroupoid is a set $H$ together with a (binary) hyperoperation. For any non-empty subsets $A, B$ of $H$, we denote

$$
A \circ B=\bigcup_{a \in A, b \in B} a \circ b
$$

Instead of $\{a\} \circ A$ and $B \circ\{a\}$, we write $a \circ A$ and $B \circ a$, respectively.

Recently, in $[14,16]$ authors introduced the notion of LA-semihypergroups as a generalization of semigroups, semihypergroups, and LA-semigroups. A hypergroupoid $(H, \circ)$ is called an LA-semihypergroup if for every $x, y, z \in H$, we have $(x \circ y) \circ z=(z \circ y) \circ x$. The law $(x \circ y) \circ z=(z \circ y) \circ x$ is called a left invertive law. An element $e \in H$ is called a left identity (resp., pure left identity) if for all $x \in H, x \in e \circ x$ (resp., $x=e \circ x)$. In an LA-semihypergroup, the medial law $(x \circ y) \circ(z \circ w)=(x \circ z) \circ(y \circ w)$ holds for all $x, y, z, w \in H$. An LA-semihypergroup may or may not contains a left identity and pure left identity. In an LA-semihypergroup $H$ with pure left identity, the paramedial law $(x \circ y) \circ(z \circ w)=(w \circ z) \circ(y \circ x)$ holds for all $x, y, z, w \in H$. If an LA-semihypergroup contains a pure left identity, then by using medial law, we get $x \circ(y \circ z)=y \circ(x \circ z)$ for all $x, y, z \in H$.

Definition 2.1. [17] Let $H$ be non-empty set and $\leq$ be an ordered relation on $H$. The triplet $(H, \circ, \leq)$ is called an ordered LA-semihypergroup if the following conditions are satisfied.

(1) $(H, \circ)$ is an LA-semihypergroup,

(2) $(H, \leq)$ is a partially ordered set,

(3) for every $a, b, c \in H, a \leq b$ implies $a \circ c \leq b \circ c$ and $c \circ a \leq c \circ b$, where $a \circ c \leq b \circ c$ means that for $x \in a \circ c$ there exist $y \in b \circ c$ such that $x \leq y$. 
Definition 2.2. [17] If $(H, \circ, \leq)$ is an ordered LA-semihypergroup and $A \subseteq H$, then $(A]$ is the subset of $H$ defined as follows:

$$
(A]=\{t \in H: t \leq a, \text { for some } a \in A\}
$$

Definition 2.3. [17] A non-empty subset $A$ of an ordered LA-semihypergroup $(H, \circ, \leq)$ is called an LAsubsemihypergroup of $H$ if $(A \circ A] \subseteq(A]$.

Definition 2.4. [17] A non-empty subset $A$ of an ordered LA-semihypergroup $(H, \circ, \leq)$ is called a right (resp., left) hyperideal of $H$ if

(1) $A \circ H \subseteq A($ resp.,$H \circ A \subseteq A)$,

(2) for every $a \in H, b \in A$ and $a \leq b$ implies $a \in A$.

If $A$ is both right hyperideal and left hyperideal of $H$, then $A$ is called a hyperideal (or two sided hyperideal) of $H$.

\section{FuZZY ordered LA-SEMIHYPERGROUPS}

Let $x \in H$, then $A_{x}=\{(y, z) \in H \circ H: x \leq y \circ z\}$. Let $f$ and $g$ be two fuzzy subsets of an ordered LA-semihypergroup $H$, then $f * g$ is defined as

$$
(f * g)(x)= \begin{cases}\bigvee_{(y, z) \in A_{x}}\{f(y) \wedge g(z)\} & \text { if } x \leq y \circ z, \text { for some } y, z \in H \\ 0 & \text { otherwise. }\end{cases}
$$

Let $F(H)$ denote the set of all fuzzy subsets of an ordered LA-semihypergroup.

Theorem 3.1. Let $H$ be an ordered LA-semihypergroup. Then the set $(F(H), *, \subseteq)$ is an ordered LAsemihypergroup.

Proof. Clearly $F(H)$ is closed. Let $f, g$ and $h$ be in $F(H)$ and let $x$ be any element of $H$ such that it is not expressible as product of two elements in $H$. Then we have,

$$
((f * g) * h)(x)=0=((h * g) * f)(x) .
$$


Let $A_{x} \neq \emptyset$. Then there exist $y$ and $z$ in $H$ such that $(y, z) \in A_{x}$. Therefore by using left invertive law, we have

$$
\begin{aligned}
((f * g) * h)(x) & =\bigvee_{(y, z) \in A_{x}}\{(f * g)(y) \wedge h(z)\} \\
& =\bigvee_{(y, z) \in A_{x}}\left\{\bigvee_{(p, q) \in A_{y}}\{f(p) \wedge g(q)\} \wedge h(z)\right\} \\
& =\bigvee_{x \leq(p \circ q) \circ z}\{f(p) \wedge g(q) \wedge h(z)\} \\
& =\bigvee_{x \leq(z \circ q) \circ p}\{h(z) \wedge g(q) \wedge f(p)\} \\
& =\bigvee_{(w, p) \in A_{x}}\left\{\bigvee_{(z, q) \in A_{w}}(h(z) \wedge g(q) \wedge f(p))\right\} \\
& =\bigvee_{(w, p) \in A_{x}}\{(h * g)(w) \wedge f(p)\}=((h * g) * f)(x)
\end{aligned}
$$

Hence $(F(H), \circ)$ is an LA-semihypergroup. Assume that $f \subseteq g$ and let $A_{x}=\emptyset$ for any $x \in H$, then

$$
(f * h)(x)=0=(g * h)(x) \Longrightarrow f * h \subseteq g * h .
$$

Similarly we can show that $f * h \supseteq g * h$. Let $A_{x} \neq \emptyset$. Then there exist $y$ and $z$ in $H$ such that $(y, z) \in A_{x}$, therefore

$$
(f * h)(x)=\bigvee_{(y, z) \in A_{x}}\{f(y) \wedge h(z)\} \leq \bigvee_{(y, z) \in A_{x}}\{g(y) \wedge h(z)\}=(g * h)(x),
$$

Similarly we can show that $f * h \supseteq g * h$. It is easy to see that $F(H)$ is a poset. Thus $(F(H), *, \subseteq)$ is an ordered LA-semihypergroup.

Theorem 3.2. Let $H$ be an ordered LA-semihypergroup. Then the property

$$
(f * g) *(h * k)=(f * h) *(g * k)
$$

holds in $F(H)$, for all $f, g, h$ and $k$ in $F(H)$.

Proof. Straightforward.

Theorem 3.3. If an ordered LA-semihypergroup $H$ has a pure left identity, then the following properties hold in $F(H)$.

(i) $(f * g) *(h * k)=(k * h) *(g * f)$,

(ii) $f *(g * h)=g *(f * h)$,

for all $f, g, h$ and $k$ in $F(H)$.

Proof. Straightforward. 
Proposition 3.1. An ordered LA-semihypergroup $(F(H), *, \subseteq)$ with $F(H)=(F(H))^{2}$ is a commutative ordered semihypergroup if and only if $(f * g) * h=f *(h * g)$ holds for all fuzzy subsets $f, g, h \in F(H)$.

Proof. Let an ordered LA-semihypergroup $F(H)$ be a commutative ordered semihypergroup. For any fuzzy subsets $f, g, h \in F(H)$, if $A_{x}=\emptyset$ for any $x \in H$, then $((f * g) * h)(x)=0=(f *(h * g))(x)$. Let $A_{x} \neq \emptyset$, then there exist $s$ and $t$ in $H$ such that $(s, t) \in A_{x}$, therefore by use of left invertive law and commutative law, we get

$$
\begin{aligned}
((f * g) * h)(x) & =\bigvee_{(s, t) \in A_{x}}\{(f * g)(s) \wedge h(t)\} \\
& =\bigvee_{(s, t) \in A_{x}}\left\{\bigvee_{(m, n) \in A_{s}} f(m) \wedge g(n) \wedge h(t)\right\} \\
& =\bigvee_{x \leq(m \circ n) \circ t}\{f(m) \wedge h(t) \wedge g(n)\} \\
& =\bigvee_{x \leq(t \circ n) \circ m}\{f(m) \wedge h(t) \wedge g(n)\} \\
& =\bigvee_{x \leq m \circ(t \circ n)}\{f(m) \wedge h(t) \wedge g(n)\} \\
& =\bigvee_{(m, p) \in A_{x}}\left\{\bigvee_{(t, n) \in A_{p}} f(m) \wedge h(t) \wedge g(n)\right\} \\
& =\bigvee_{(m, p) \in A_{x}}\{f(m) \wedge(h * g)(p)\} \\
& =\underset{(f *(h * g))(x) .}{ }
\end{aligned}
$$

Thus $(f * g) * h=f *(h * g)$. Conversely, let $(f * g) * h=f *(h * g)$ holds for all fuzzy subsets $f, g, h \in F(H)$. We have to show that $H$ is a commutative ordered semihypergroup. Let $f$ and $g$ be any arbitrary fuzzy subsets of $H$, if $A_{x}=\emptyset$ for any $x \in H$, then $(f * g)(x)=0=(g * f)(x)$. Let $A_{x} \neq \emptyset$, then there exist $s$ and $t$ in $H$ such that $(s, t) \in A_{x}$. Since $F(H)=(F(H))^{2}$, so $f=h * k$, where $h$ and $k$ are any fuzzy subsets of $H$. Now by left invertive law, we have

$$
\begin{aligned}
(f * g)(x) & =((h * k) * g)(x)=\bigvee_{(s, t) \in A_{x}}\{(h * k)(s) \wedge g(t)\} \\
& =\bigvee_{(s, t) \in A_{x}}\left\{\bigvee_{(m, n) \in A_{s}} h(m) \wedge k(n) \wedge g(t)\right\} \\
& =\bigvee_{x \leq(m \circ n) \circ t}\{g(t) \wedge k(n) \wedge h(m)\} \\
& =\bigvee_{x \leq(t \circ n) \circ m}\{g(t) \wedge k(n) \wedge h(m)\}
\end{aligned}
$$




$$
\begin{aligned}
& =\bigvee_{(p, m) \in A_{x}}\left\{\bigvee_{(t, n) \in A_{p}} g(t) \wedge k(n) \wedge h(m)\right\} \\
& =\bigvee_{(p, m) \in A_{x}}\{(g * k)(p) \wedge h(m)\}=(g *(h * k))(x) \\
& =(g * f)(x) .
\end{aligned}
$$

This shows that $f * g=g *(h * k)=g * f$. Therefore commutative law holds in $F(H)$.

Now if $A_{x}=\emptyset$ for any $x \in H$, then $((f * g) * k)(x)=0=(f *(g * k))(x)$. Let $A_{x} \neq \emptyset$, then there exist $s$ and $t$ in $H$ such that $(s, t) \in A_{x}$, therefore by using left invertive law and commutative law, we get

$$
\begin{aligned}
((f * g) * k)(x) & =\bigvee_{(s, t) \in A_{x}}\{(f * g)(s) \wedge k(t)\} \\
& =\bigvee_{(s, t) \in A_{x}}\left\{\bigvee_{(m, n) \in A_{s}} f(m) \wedge g(n) \wedge k(t)\right\} \\
& =\bigvee_{x \leq(m \circ n) \circ t}\{f(m) \wedge g(n) \wedge k(t)\} \\
& =\bigvee_{x \leq(t \circ n) \circ m}\{f(m) \wedge g(n) \wedge k(t)\} \\
& =\bigvee_{x \leq m \circ(t \circ n)}\{f(m) \wedge g(n) \wedge k(t)\} \\
& =\bigvee_{x \leq m \circ(n \circ t)}\{f(m) \wedge g(n) \wedge k(t)\} \\
& =\bigvee_{(m, p) \in A_{x}}\left\{\underset{(n, t) \in A_{p}}{\bigvee} f(m) \wedge g(n) \wedge k(t)\right\} \\
& =\bigvee_{(m, p) \in A_{x}}\{f(m) \wedge(g * k)(p)\} \\
& =(f *(g * k)(x) .
\end{aligned}
$$

Therefore associative law holds in $F(H)$. Thus $F(H)$ is commutative ordered semihypergroup.

Theorem 3.4. $\mathcal{C}_{\mathcal{M}}=\{f \mid f \in F(H), f * \alpha=f$, where $\alpha=\alpha * \alpha\}$ is a commutative monoid in $H$.

Proof. The fuzzy subset $\mathcal{C}_{\mathcal{M}}$ of $H$ is non-empty since $\alpha * \alpha=\alpha$, which implies that $\alpha$ is in $\mathcal{C}_{\mathcal{M}}$. Let $f$ and $\gamma$ be fuzzy subsets of $H$ in $\mathcal{C}_{\mathcal{M}}$, then $f * \alpha=f$ and $\gamma * \alpha=\gamma$. If $A_{x}=\emptyset$ for $x \in H$, then $(f * \gamma)(x)=0=((f * \gamma) * \alpha)(x)$. Let $A_{x} \neq \emptyset$, then there exist $y$ and $z$ in $H$ such that $(y, z) \in A_{x}$. Therefore 
by using medial law, we have

$$
\begin{aligned}
(f * \gamma)(x) & =\bigvee_{(y, z) \in A_{x}}\{(f * \alpha)(y) \wedge(\gamma * \alpha)(z)\} \\
& =\bigvee_{(y, z) \in A_{x}}\left\{\bigvee_{(p, q) \in A_{y}}\{f(p) \wedge \alpha(q)\} \wedge \bigvee_{(u, v) \in A_{z}}\{\gamma(u) \wedge \alpha(v)\}\right\} \\
& =\bigvee_{x \leq(p \circ q) \circ(u \circ v)}\{f(p) \wedge \alpha(q) \wedge \gamma(u) \wedge \alpha(v)\} \\
& =\bigvee_{x \leq(p \circ u) \circ(q \circ v)}\{f(p) \wedge \gamma(u) \wedge \alpha(q) \wedge \alpha(v)\} \\
& =\bigvee_{(m, n) \in A_{x}}\left\{\bigvee_{(p, u) \in A_{m}}\{f(p) \wedge \gamma(u)\} \wedge \bigvee_{(q, v) \in A_{n}}\{\alpha(q) \wedge \alpha(v)\}\right\} \\
& =\bigvee_{(m, n) \in A_{x}}\{(f * \gamma)(m) \wedge(\alpha * \alpha)(n)\}=((f * \gamma) *(\alpha * \alpha))(x) .
\end{aligned}
$$

Thus $f * \gamma=(f * \gamma) *(\alpha * \alpha)=(f * \gamma) * \alpha$, which implies that $\mathcal{C}_{\mathcal{M}}$ is closed.

Now if $A_{x}=\emptyset$ for $x \in H$, then $(f * \gamma)(x)=0=(\gamma * f)(x)$. Let $A_{x} \neq \emptyset$, then there exist $y$ and $z$ in $H$ such that $(y, z) \in A_{x}$. Therefore by using left invertive law, we have

$$
\begin{aligned}
(f * \gamma)(x) & =\bigvee_{(y, z) \in A_{x}}\{(f * \alpha)(y) \wedge \gamma(z)\} \\
& =\bigvee_{(y, z) \in A_{x}}\left\{\bigvee_{(p, q) \in A_{y}} f(p) \wedge \alpha(q) \wedge \gamma(z)\right\} \\
& =\bigvee_{x \leq(p \circ q) \circ z}\{\gamma(z) \wedge \alpha(q) \wedge f(p)\} \\
& =\bigvee_{x \leq(z \circ q) \circ p}\{\gamma(z) \wedge \alpha(q) \wedge f(p)\} \\
& =\bigvee_{(t, p) \in A_{x}}\left\{\bigvee_{(z, q) \in A_{t}} \gamma(z) \wedge \alpha(q) \wedge f(p)\right\} \\
& =\bigvee_{(t, p) \in A_{x}}\{(\gamma * \alpha)(t) \wedge f(p)\}=((\gamma * \alpha) * f)(x)
\end{aligned}
$$

Thus $f * \gamma=(\gamma * \alpha) * f=\gamma * f$, which implies that commutative law holds in $\mathcal{C}_{\mathcal{M}}$ and associative law holds in $\mathcal{C}_{\mathcal{M}}$ due to commutativity. Since for any fuzzy subset $f$ in $\mathcal{C}_{\mathcal{M}}$, we have $f * \alpha=f$ (where $\alpha$ is fixed) implies that $\alpha$ is a right identity in $H$ and hence an identity.

For an ordered LA-semihypergroup $H$, the fuzzy subset $\mathbf{H}$ of $H$ is defined as follows:

$$
\mathbf{H}: H \rightarrow[0,1] \mid x \rightarrow \mathbf{H}(x):=1
$$

Lemma 3.1. In an ordered LA-semihypergroup with a left identity $\mathbf{H} * \mathbf{H}=\mathbf{H}$. 
Proof. Let $x \in H$. Then $x \leq e \circ x$, that is $(e, x) \in A_{x}$, where $e$ is the left identity of $H$. Therefore

$$
(\mathbf{H} * \mathbf{H})(x)=\bigvee_{(e, x) \in A_{x}}(\mathbf{H}(e) \wedge \mathbf{H}(x))=1=\mathbf{H}(x) .
$$

So $\mathbf{H} * \mathbf{H}=\mathbf{H}$.

\section{FUZZY HYPERIDEALS IN ORDERED LA-SEMIHYPERGROUPS}

In this section, we define the concept of a fuzzy right (resp., left) hyperideal and give relationships between them.

Definition 4.1. Let $(H, \circ, \leq)$ be an ordered LA-semihypergroup. A fuzzy subset $f: H \rightarrow[0,1]$ is called fuzzy LA-subsemihypergroup of $H$ if the following assertion are satisfied;

(i) $\bigwedge_{z<a \circ b} f(z) \geq \min \{f(a), f(b)\}$,

(ii) if $a \leq b$ implies $f(a) \geq f(b)$,

for every $a, b \in H$.

Example 4.1. We consider a set $H=\{x, y, z\}$ with the following hyperoperation " $\circ$ " and the order " $\leq$ ":

\begin{tabular}{|l|l|l|l|}
\hline$\circ$ & $x$ & $y$ & $z$ \\
\hline$x$ & $\{x, y\}$ & $\{x, y\}$ & $z$ \\
\hline$y$ & $\{x, z\}$ & $\{x, z\}$ & $z$ \\
\hline$z$ & $z$ & $z$ & $z$ \\
\hline
\end{tabular}

$$
\leq:=\{(x, x),(y, y),(z, x),(z, y),(z, z)\}
$$

We give the covering relation " $\prec "$ and the figure of $H$ as follows:

$$
\prec=\{(z, x),(z, y)\} .
$$

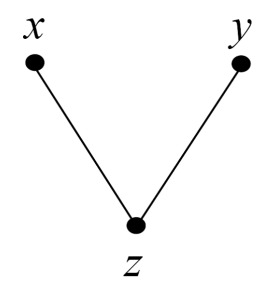

Then $(H, \circ, \leq)$ is an ordered LA-semihypergroup. Now let $f$ be a fuzzy subset of $H$ such that

$$
f(x)=0.5, f(y)=0.3, f(z)=0.9 .
$$

Clearly $f$ is a fuzzy LA-subsemihypergroup of $H$. 
Theorem 4.1. A fuzzy subset $f$ of an ordered LA-semihypergroup $H$ is a fuzzy LA-subsemihypergroup of $H$ if and only if

(i) $f * f \subseteq f$,

(ii) if $a \leq b$ implies $f(a) \geq f(b)$, for every $a, b \in H$.

Proof. Consider that $f$ is a fuzzy LA-subsemihypergroup of $H$. Let $A_{x}=\emptyset$ for any $x \in H$. Then $(f * f)(x)=$ $0=f(x)$. Let $A_{x} \neq \emptyset$. Then for $x, y, a \in H$, we have

$$
(f * f)(a)=\bigvee_{a \leq x \circ y}\{f(x) \wedge f(y)\} \leq \bigvee_{a \leq x \circ y} f(a)=f(a)
$$

Thus $f * f \subseteq f$.

Conversely, assume that $f * f \subseteq f$. Let $x, y, a \in H$ with $a \in x \circ y$. We have

$$
f(a) \geq(f * f)(a)=\bigvee_{a \leq x \circ y}\{f(x) \wedge f(y)\} \geq f(x) \wedge f(y) .
$$

Thus $\bigwedge_{a \leq x \circ y} f(a) \geq \min \{f(x), f(y)\}$. Thus $f$ is a fuzzy LA-subsemihypergroup of $H$.

Definition 4.2. Let $(H, \circ, \leq)$ be an ordered LA-semihypergroup. A fuzzy subset $f: H \rightarrow[0,1]$ is called a fuzzy right (resp., left) hyperideal of $H$ if

(1) $\bigwedge_{z \leq a \circ b} f(z) \geq f(a)$ (resp., $\left.\bigwedge_{z \leq a \circ b} f(z) \geq f(b)\right)$,

(2) $a \leq b$ implies $f(a) \geq f(b)$,

for every $a, b \in H$.

If $f$ is both fuzzy right hyperideal and fuzzy left hyperideal of $H$, then $f$ is called a fuzzy hyperideal of H.

Definition 4.3. We consider a set $H=\{x, y, z\}$ with the following hyperoperation " $"$ and the order " $\leq$ ":

\begin{tabular}{|c|c|c|c|c|}
\hline$\circ$ & $x$ & $y$ & $z$ & $w$ \\
\hline$x$ & $x$ & $\{x, w\}$ & $\{x, w\}$ & $w$ \\
\hline$y$ & $\{x, w\}$ & $\{y, z\}$ & $\{y, z\}$ & $w$ \\
\hline$z$ & $\{x, w\}$ & $y$ & $y$ & $w$ \\
\hline$w$ & $w$ & $w$ & $w$ & $w$ \\
\hline
\end{tabular}

$$
\leq:=\{(x, x),(x, y),(x, z),(y, y),(z, z),(w, x),(w, y),(w, z),(w, w)\}
$$

We give the covering relation " $\prec "$ and the figure of $H$ as follows:

$$
\prec=\{(x, y),(x, z),(w, x)\}
$$




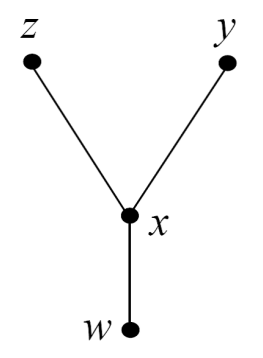

Then $(H, \circ, \leq)$ is an ordered LA-semihypergroup. Now let $f$ be a fuzzy subset of $H$ such that

$$
f(a)=\left\{\begin{array}{c}
0.6 \text { if } a=x \\
0.4 \text { if } a=y \\
0.2 \text { if } a=z \\
0.9 \text { if } a=w
\end{array}\right.
$$

Then $f$ is a fuzzy two sided hyperideal of $H$.

Theorem 4.2. A fuzzy subset $f$ of an ordered LA-semihypergroup $H$ is a fuzzy left (resp., right) hyperideal of $H$ if and only if

(i) $\mathbf{H} * f \subseteq f(r e s p ., f * \mathbf{H} \subseteq f)$

(ii) if $a \leq b$ implies $f(a) \geq f(b)$, for every $a, b \in H$.

Proof. The proof is similar to the proof of the Theorem 4.1 .

Definition 4.4. Let $H$ be an ordered LA-semihypergroup and $f$ be a fuzzy subset of $H$. Then for every $t \in[0,1]$ the set

$$
f_{t}=\{x: x \in H, f(x) \geq t\}
$$

is called the level set of $H$.

Definition 4.5. Let $H$ be an ordered LA-semihypergroup and $\emptyset \neq A \subseteq H$. Then the characteristic function $\chi_{A}$ of $A$ is defined as:

$$
\chi_{A}: H \rightarrow[0,1]: x \rightarrow \chi_{A}(x)= \begin{cases}1 & \text { if } x \in A \\ 0 & \text { if } x \notin A .\end{cases}
$$

Theorem 4.3. Let $H$ be an ordered LA-semihypergroup and $f$ be a fuzzy subset of $H$. Then $f$ is a fuzzy LA-subsemihypergroup (resp., right hyperideal, left hyperideal) of $H$ if and only if for every $t \in[0,1]$, the non-empty level subset $f_{t}$ is a fuzzy LA-subsemihypergroup (resp., right hyperideal, left hyperideal) of $H$.

Proof. Assume that $f$ is a fuzzy right hyperideal of $H$. Let $t \in[0,1]$ with $f_{t} \neq \phi$. Let $a \in f_{t} \circ H$. We have $a \in b \circ h$ for some $b \in f_{t}$ and $h \in H$. By assumption, $t \leq f(b) \leq \bigwedge_{a \in b \circ h} f(a)$, we have $f(a) \geq t$. This implies 
$f_{t} \circ H \subseteq f_{t}$. Let $x \in f_{t}$ and $y \in H$ with $y \leq x$. Since $t \leq f(x) \leq f(y)$, we obtain $y \in f_{t}$. Therefore, $f_{t}$ is right hyperideal of $H$.

Conversely, we assume that for every $t \in[0,1], f_{t}$ is a right hyperideal of $H$. We show that $f_{a} \leq \bigwedge_{c \in a \circ b} f(c)$ for $a, b \in H$. We put $t_{\circ}=f(a)$. By assumption $f_{t_{\circ}}$ is a right hyperideal of $H$. Since $a \in f_{t_{\circ}}, a \circ b \subseteq f_{t_{\circ}}$. Then, for every $c \in a \circ b$, we obtain $t_{\circ} \leq f(c)$ and hence, $f(a)=t_{\circ} \leq \bigwedge_{c \in a \circ b} f(c)$. Let $a, b \in H$ with $a \leq b$. Since $a \leq b, b \in f_{f(b)}$ and $f_{f(b)}$ is a right hyperideal of $H$. We have $a \in f_{f_{b}}$. So, $f(b) \leq f(a)$. Therefore $f$ is a fuzzy right hyperideal of $H$.

Corollary 4.1. Let $H$ be an ordered LA-semihypergroup and $\chi_{I}$ be the characteristic function of I. Then, then $I$ is an LA-subsemihypergroup (resp., right hyperideal, left hyperideal) of $H$ if and only if $\chi_{I}$ is a fuzzy LA-subsemihypergroup (resp., right hyperideal, left hyperideal) of $H$.

Theorem 4.4. If $\left\{f_{i}\right\}_{i \in J}$ is a family of fuzzy left hyperideals (resp., right hyperideals) of an ordered LAsemihypergroup $H$, then $\bigcap_{i \in J} f_{i}$ is a fuzzy left hyperideal (resp., right hyperideal) of $H$, where

$$
\bigcap_{i \in J} f_{i}=\bigwedge_{i \in J} f_{i} \text { and } \bigwedge_{i \in J} f_{i}(x)=\inf \left\{f_{i}(x): i \in J, x \in H\right\}
$$

Proof. Straightforward.

Proposition 4.1. The fuzzy product of two fuzzy right (resp., left) hyperideals of an ordered LA-semihypergroup $H$ is again a fuzzy right (resp., left) hyperideal of $H$.

Proof. Let $f_{1}$ and $f_{2}$ be two fuzzy right hyperideals of an ordered LA-semihypergroup $H$. Let $x, y \in H$ such that $x \leq y$. Let $(a, b) \in A_{y}$ then $y \leq a \circ b$. Since $x \leq y$, so $x \leq a \circ b$ implies $(a, b) \in A_{x}$. Hence $A_{y} \subseteq A_{x}$. Now

$$
\begin{aligned}
\left(f_{1} * f_{2}\right)(y) & =\bigvee_{(a, b) \in A_{y}}\left\{f_{1}(a) \wedge f_{2}(b)\right\}=\bigvee_{(a, b) \in A_{y} \subseteq A_{x}}\left\{f_{1}(a) \wedge f_{2}(b)\right\} \\
& \leq \bigvee_{(a, b) \in A_{x}}\left\{f_{1}(a) \wedge f_{2}(b)\right\} \\
& =\left(f_{1} * f_{2}\right)(x)
\end{aligned}
$$

Thus $\left(f_{1} * f_{2}\right)(x) \geq\left(f_{1} * f_{2}\right)(y)$. If $A_{x}=\emptyset$, for any $x \in H$. Then

$$
\bigwedge_{x \leq a \circ b}\left(f_{1} * f_{2}\right)(x)=0=\left(f_{1} * f_{2}\right)(a)
$$


If $A_{x} \neq \emptyset$. Since $f_{1}$ and $f_{2}$ are a fuzzy right hyperideals of $H$, then

$$
\begin{aligned}
\left(f_{1} * f_{2}\right)(x) & =\bigvee_{(a, b) \in A_{x}}\left\{f_{1}(a) \wedge f_{2}(b)\right\} \\
& \leq \bigvee_{(a \circ y, b \circ y) \in A_{x \circ y}}\left\{\left(\bigwedge_{m \leq a \circ y} f_{1}(m)\right) \wedge\left(\bigwedge_{n \leq b \circ y} f_{2}(n)\right)\right\} \\
& =\bigwedge_{q \leq x \circ y}\left(f_{1} * f_{2}\right)(q) .
\end{aligned}
$$

Thus $f_{1} * f_{2}$ is a fuzzy right hyperideal of $H$.

Theorem 4.5. Let $f_{1}$ be a fuzzy right hyperideal and $f_{2}$ a fuzzy left hyperideal of $H$. Then $f_{1} * f_{2} \subseteq f_{1} \cap f_{2}$.

Proof. Let $A_{x}=\emptyset$ for any $x \in H$. Then $\left(f_{1} * f_{2}\right)(x)=0=\left(f_{1} \cap f_{2}\right)(x)$. Given that $f_{1}$ is a fuzzy right hyperideal of $H$, i.e. $\bigwedge_{a \leq x \circ y} f_{1}(a) \geq f_{1}(x)$ also given that $f_{2}$ is fuzzy left hyperideal of $H$, i.e. $\bigwedge_{a \leq x \circ y} f_{2}(a) \geq$ $f_{2}(y)$. Let $A_{x} \neq \emptyset$. Then for $x, y, a \in H$, we have

$$
\begin{aligned}
\left(f_{1} * f_{2}\right)(a) & =\bigvee_{a \leq x \circ y}\left\{f_{1}(x) \wedge f_{2}(y)\right\} \leq \bigvee_{a \leq x \circ y}\left\{\bigwedge_{a \leq x \circ y} f_{1}(a) \wedge \bigwedge_{a \leq x \circ y} f_{2}(a)\right\} \\
& =\left(f_{1} \cap f_{2}\right)(a) .
\end{aligned}
$$

Thus $f_{1} * f_{2} \subseteq f_{1} \cap f_{2}$.

Lemma 4.1. Let $H$ be an ordered LA-semihypergroup with left identity. Then every fuzzy right hyperideal of $H$ is fuzzy left hyperideal of $H$.

Proof. Let $H$ be an LA-semihypergroup with pure left identity $e$, and $f$ be a fuzzy right hyperideal of $H$. Since $f$ is a fuzzy right hyperideal of $H$, so $f * \mathbf{H} \subseteq f$. Thus by Lemma 3.1, and left invertive law, we have

$$
\mathbf{H} * f=(\mathbf{H} * \mathbf{H}) * f=(f * \mathbf{H}) * \mathbf{H} \subseteq f * \mathbf{H} \subseteq f .
$$

Thus $\mathbf{H} * f \subseteq f$. Hence $f$ is a fuzzy left hyperideal of $H$.

Theorem 4.6. If $f$ is a fuzzy left hyperideal of $H$ with left identity, then $f \cup(f * \mathbf{H})$ is a fuzzy hyperideal of $H$.

Proof. Let $f$ be a fuzzy left hyperideal of $H$. We have to show that $f \cup(f * \mathbf{H})$ is fuzzy hyperideal. Let

$$
\begin{aligned}
(f \cup(f * \mathbf{H})) * \mathbf{H} & =(f * \mathbf{H}) \cup(f * \mathbf{H}) * \mathbf{H} \\
& =(f * \mathbf{H}) \cup(\mathbf{H} * \mathbf{H}) * f \\
& =(f * \mathbf{H}) \cup(\mathbf{H} * f) \\
& \subseteq(f * \mathbf{H}) \cup f \\
& =f \cup(f * \mathbf{H}) .
\end{aligned}
$$


Hence $f \cup(f * \mathbf{H})$ is fuzzy right hyperideal of $H$. Since every fuzzy right hyperideal of an ordered LAsemihypergroup with left identity is a fuzzy left hyperideal of $H$, so $f \cup(f * \mathbf{H})$ is a fuzzy hyperideal of $H$.

Definition 4.6. A fuzzy hyperideal $f$ of an ordered LA-semihypergroup $H$ is called idempotent if

$$
f * f=f .
$$

Proposition 4.2. Every idempotent fuzzy left hyperideal of an ordered LA-semihypergroup $H$ is a fuzzy hyperideal of $H$.

Proof. Let $f$ be a fuzzy left hyperideal of $H$ which is idempotent. Then

$$
f * \mathbf{H}=(f * f) * \mathbf{H}=(\mathbf{H} * f) * f \subseteq f * f=f .
$$

Hence $f$ is a fuzzy right hyperideal of $H$ and so $f$ is a fuzzy hyperideal of $H$.

Proposition 4.3. If $f$ is an idempotent fuzzy set in an ordered LA-semihypergroup $H$ with left identity. Then $\mathbf{H} * f$ and $f * \mathbf{H}$ are idempotents.

Proof. Let $f$ be an idempotent element in an ordered LA-semihypergroup $H$ with left identity. Then by using medial law, we have

$$
(\mathbf{H} * f) *(\mathbf{H} * f)=(\mathbf{H} * \mathbf{H}) *(f * f)=\mathbf{H} * f .
$$

Thus $(\mathbf{H} * f) *(\mathbf{H} * f)=\mathbf{H} * f$. The case for $f * \mathbf{H}$ can be seen in a similar way.

\section{Conclusion}

Fuzzy set theory is a mathematical tools for dealing with uncertainties. This paper is devoted to the discussion of the combinations of fuzzy set in ordered LA-semihypergroup. We combined these concepts to introduce fuzzy left (resp., right) hyperideals and discussed some interesting results.

\section{REFERENCES}

[1] F. Marty, Sur uni generalization de la notion de groupe, 8th Congress Math. Scandinaves, Stockholm (1934) 45-49.

[2] P. Bonansinga and P. Corsini, On semihypergroup and hypergroup homomorphisms, Boll. Un. Mat. Ital. B., 1(2) (1982) 717-727.

[3] B. Davvaz, Some results on congruences in semihypergroups, Bull. Malyas. Math. Sci. So. 23 (2000) 53-58.

[4] D. Freni, Minimal order semihypergroups of type U on the right, II, J. Algebra, 340 (2011) 77-80.

[5] K. Hilla, B. Davvaz and K. Naka, On quasi-hyperideals in semihypergroups, Commun. Algebra, 39 (2011) 4183.

[6] V. Leoreanu, About the simplifiable cyclic semihypergroups, Italian J. Pure Appl. Math., 7 (2000) 69-76.

[7] M. D. Salvo, D. Freni and G. Lo Faro, Fully simple semihypergroups, J. Algebra, 399 (2014) 358-377.

[8] D. Heidari and B. Davvaz, On ordered hyperstructures, U. P. B. Sci. Bull. Series A, 73 (2011) 85-96. 
[9] M. Bakhshi and R.A. Borzooei, Ordered polygroups, Ratio Math, 24 (2013) 31-40.

[10] J. Chvalina, Commutative hypergroups in the sense of Marty and ordered sets, in: Proc. Summer School, Gen. Algebra ordered Sets, Olomouc (Czech Republic), (1994) 19-30.

[11] S. Hoskova, Upper order hypergroups as a reflective subcategory of subquasiorder hypergroups, Ital. J. Pure Appl. Math., 20 (2006) 2015-222.

[12] M. Kondo and N. Lekkoksung, On intra-regular ordered Г-semihypergroups, Int. J. Math. Anal., 7(28) (2013) $1379-1386$.

[13] M. Novak, EL-hyperstructures, Ratio Math., 23 (2012) 65-80.

[14] K. Hila and J. Dine, On hyperideals on left almost semihypergroups, ISRN Algebra, (2011) Article ID 9531248 Pages.

[15] M. A. Kazim and M. Naseerudin, On almost semigroup, Aligarh Bull. Math., 2 (1972) 1-7.

[16] N. Yaqoob, P. Corsini and F. Yousafzai, On Intra-regular left almost semihypergroups with pure left identity, J. Math., Article ID 510790 (2013) 10 pages.

[17] N. Yaqoob and M. Gulistan, Partially ordered left almost semihypergroups, J. Egyptian Math. Soc., 23(2) (2015) $231-235$.

[18] L. A. Zadeh, Fuzzy sets, Inform. Control., 8 (1965) 338-353.

[19] A. Rosenfeld, Fuzzy groups, J. Math. Anal. Appl., 35 (1971) 512-517.

[20] P. Corsini, M. Shabir and T. Mahmood, Semisimple semihypergroups in terms of hyperideals, Iran J. Fuzzy. Syst., 8 (2011) 95-111.

[21] P. Corsini, Fuzzy multiset hyperstructures, European J. Comb., 44 (2015) 198-207.

[22] B. Davvaz, Characterization of subsemihypergroups by various triangular norms, Czech. Math. J., 55(4) (2005) 923-932.

[23] B. Davvaz, Fuzzy hyperideals in semihypergroups, Italian J. Pure Appl. Math., 8 (200) 67-74.

[24] K. Hila and S. Abdullah, A study on intuitionistic fuzzy sets in Г-semihypergroups, J. Intell. Fuzzy Syst., 26 (2014) 1695-1710.

[25] A. Khan, M. Farooq, M. Izhar and B. Davvaz, Fuzzy hyperideals of left almost semihypergroups, Int. J. Anal. Appl., 15(2) (2017) 155-171.

[26] B. Pibaljommee, K. Wannatong and B. Davvaz, An investigation on fuzzy hyperideals in ordered in semihypergroups, Quasigroups and Relat. Syst., 23 (2015) 297-308.

[27] B. Pibaljommee and B. Davvaz, Characterizations of (fuzzy) bi-hyperideals in ordered semihypergroups, J. Intell. Fuzzy Syst., 28 (2015) 2141-2148.

[28] J. Tang, A. Khan and Y. Luo, Characterizations of semisimple ordered semihypergroups in term of fuzzy hyperideals, J. Intell. Fuzzy Syst., 30(3) (2016) 1735-1753.

[29] J. Tang, B. Davvaz and X. Xie, A study on (fuzzy) quasi- $\Gamma$-hyperideals in ordered $\Gamma$-semihypergroups, J. Intell. Fuzzy Syst., 32(6) (2017) 3821-3838.

[30] J. Tang, B. Davvaz and Y. Luo, A study on fuzzy hyperideals in ordered semihypergroups, Italian J. Pure Appl. Math., 36 (2016) 125-146.

[31] J. Tang, B. Davvaz and Y.F. Luo, Hyperfilters and fuzzy hyperfilters of ordered semihypergroups, J. Intell. Fuzzy Syst., 29(1) (2015) 75-84.

[32] N. Tipachot and B. Pibaljommee, Fuzzy interior hyperideasl in ordered semihypergroups, Italian J. Pure Appl. Math., 36 (2016) 859-870.

[33] J. Zhan and B. Davvaz, Study of fuzzy algebraic hypersystems from a general view point, Int. J. Fuzzy Syst., 12 (2010) 73-79.

[34] J. Zhan, B. Davvaz and K.P. Shum, A new view of fuzzy hypernear-rings, Inform Sci., 178 (2008) 425-438. 